\title{
Efeitos dos Controles por Seleção e por Rejeição sobre os Movimentos do Olhos*
}

\author{
William F. Perez ${ }^{1,2,3, * *}$ (1) \& Gerson Y. Tomanari ${ }^{1,2}$ (1) \\ ${ }^{1}$ Universidade de São Paulo, São Paulo, SP, Brasil \\ ${ }^{2}$ Instituto Nacional de Ciência e Tecnologia sobre Comportamento, Cognição e Ensino \\ (INCT-ECCE), São Carlos, SP, Brasil \\ ${ }^{3}$ Paradigma - Centro de Ciências e Tecnologia do Comportamento, São Paulo, SP, Brasil
}

\begin{abstract}
RESUMO - O presente estudo avaliou o efeito dos controles por seleção e por rejeição sobre a observação de $\mathrm{S}+\mathrm{e} \mathrm{S}-\mathrm{em}$ treinos de emparelhamento com o modelo. Quatro adultos foram expostos a treinos de discriminação condicional e testes de equivalência. Na condição de Linha de Base (LB), nenhuma relação de controle modelo/comparação específica foi favorecida; nas condições de Controle por Rejeição e Controle por Seleção, as relações modelo/S- e modelo/S+ foram favorecidas, respectivamente. Na condição LB, foi verificada uma maior observação do S+ em comparação ao S-. O estabelecimento do controle por rejeição tendeu a ser acompanhado por uma maior observação do S- e por escolhas baseadas na observação exclusiva do S-; no caso do controle por seleção, padrões similares foram verificados, porém para o S+. Foi concluído que o estabelecimento dos controles por seleção e por rejeição são acompanhados de mudanças na observação dos estímulos de comparação.
\end{abstract}

PALAVRAS-CHAVE: equivalência de estímulos, controle por seleção, controle por rejeição, emparelhamento com o modelo, movimentos dos olhos

\section{Effects of Select and Reject Controls on Eye Movements}

\begin{abstract}
The present study investigated the effects of select and reject controls upon $\mathrm{S}+$ and $\mathrm{S}$ - observations during a matching-to-sample training. Four adults were exposed to discriminative trainings and equivalence tests. In Baseline (BL), no specific sample-comparison control was biased; in the Reject Control and Select Control conditions, sample/S+ and sample/S- relations were biased, respectively. In BL, participants observed more the S + than the S-. The establishment of reject control tended to be followed by an increase in the observation of the S- and by choices based on the exclusive observation of the S-; in the case of select control, similar patterns were found, however, for the S+. Thus, the establishment of select and reject controls are followed by changes in the observation of comparison stimuli.
\end{abstract}

KEYWORDS: stimulus equivalence, select control, reject control, matching-to-sample, eye movements

O procedimento de Matching to Sample ou pareamento ao modelo (MTS) tem sido tradicionalmente utilizado para o estudo de relações simbólicas entre estímulos (Sidman, 1994; ver também de Rose \& Bortoloti, 2007). Nesse procedimento, um estímulo modelo é apresentado simultaneamente a dois ou mais estímulos de comparação. Respostas de escolha dos estímulos de comparação arbitrariamente designados como "corretos" ( $\mathrm{S}+$ ) são seguidas de feedback de acertos; respostas de escolha dos estímulos de comparação arbitrariamente designados como "incorretos" (S-) são seguidas de feedbacks de erro ou, simplesmente, pelo início da próxima tentativa. Em uma tarefa de MTS com dois estímulos de comparação, a escolha do estímulo designado como correto, no entanto, pode ocorrer sob controle de pelo menos duas relações modelocomparação: o participante pode escolher o estímulo correto

\footnotetext{
* Apoio: CNPq (Processo No. 573972/2008-7 e 465686/2014-1) e FAPESP (Processo 2014/50909-8 e 08/57705-8). Bolsa de Mestrado concedida ao primeiro autor FAPESP (processo: 06/05165-4).

** E-mail: will.f.perez@gmail.com

- Submetido: 30/03/2017; Revisado: 13/04/2018; Aceito: 18/07/2028.
} 
sob controle do modelo e do $\mathrm{S}+$ apresentado (controle por seleção) ou sob controle do modelo e do S- (controle por rejeição). Em ambos os casos, uma resposta correta é registrada, porém o controle de estímulos é distinto. No primeiro caso, o estímulo correto é escolhido a partir da seleção do estímulo correto, enquanto que, no segundo caso, o estímulo correto é escolhido a partir da rejeição do estímulo incorreto (e.g., Carrigan \& Sidman, 1992; Johnson $\&$ Sidman, 1993).

O ensino de relações condicionais pelo procedimento de MTS, quando realizado com humanos verbalmente competentes, frequentemente dá origem a relações de equivalência. A equivalência entre os estímulos relacionados é demonstrada quando o participante, depois de aprender um conjunto inicial de relações condicionais (e.g., AB e $\mathrm{BC}$ ), responde a novas relações originadas a partir de recombinações das relações apresentadas durante o treino (e.g., AC, CA, BA, CB, AA, BB, CC). Quando as respostas às novas relações ocorrem em concordância com as propriedades de transitividade (AC), simetria (BA e $\mathrm{CB}$ ) e reflexividade (AA, BB e CC), atesta-se a formação de classes de equivalência (Sidman, 1994; Sidman \& Tailby, 1982).

As ocorrências de resultados negativos nos testes de formação de classes de estímulos equivalentes, após a realização de treinos de discriminação condicional bemsucedidos, indicam a possibilidade de que outras variáveis, além da aprendizagem de um conjunto inicial de relações condicionais, estejam envolvidas no estabelecimento de relações de equivalência (de Rose, 1996; Mcllvane et al., $\&$ Stromer, 2000). Dentre essas variáveis está o tipo de relação modelo-comparação estabelecido durante o treino, que determina a resposta de escolha de um dos estímulos de comparação, ou seja, o controle por seleção ou por rejeição (Carrigan \& Sidman, 1992; Johnson \& Sidman, 1993; Perez et al., 2015, 2017, 2020). Enquanto o estabelecimento do controle por seleção (e.g., A1 - seleção de B1; B1 - seleção de $\mathrm{C} 1$ ) é relacionado à ocorrência de altos índices de acerto nos testes de equivalência (e.g., transitividade: A1 - seleção de $\mathrm{C} 1$; simetria: $\mathrm{B} 1$ - seleção de $\mathrm{C} 1, \mathrm{C} 1$ - seleção de $\mathrm{B} 1$; reflexividade: A1 - seleção de A1), o estabelecimento do controle por rejeição, de maneira uniforme para todas as relações (e.g., A1 - rejeição de B2, B2 - rejeição de C1), está relacionado a escores próximos a zero nos testes de transitividade (e.g., $\mathrm{A} 1$ - rejeição de $\mathrm{C} 1$, seleção de $\mathrm{C} 2$ ), equivalência (e.g., C1 - rejeição de A1, seleção de A2) e reflexividade (e.g., A1 - rejeição de A1, seleção de A2; para uma explicação detalhada, ver Carrigan \& Sidman, 1992; Johnson \& Sidman, 1993; Perez \& Tomanari, 2008, 2013; Perez et al., 2015, 2017, 2020). Os testes de simetria, nesse caso, não são afetados pelo controle por rejeição (e.g., A1 -rejeição de B2, escolha de B1; para um explicação detalhada, ver Carrigan \& Sidman, 1992; em português, ver Perez \& Tomanari, 2008, 2013).

Durante o treino de discriminação condicional por meio do procedimento de MTS, tal como tem sido tradicionalmente empregado na literatura (Sidman, 1994), pouco pode ser dito acerca dos controles por seleção ou por rejeição. Para tanto, faz-se necessário realizar, posteriormente ao treino das relações condicionais iniciais, testes que indiquem o tipo de relação modelo-comparação estabelecida (para uma revisão, ver Perez \& Tomanari, 2013). Tais testes envolvem, por exemplo, substituir um dos estímulos de comparação por estímulos novos (Dixon \& Dixon, 1978; Huziwara et al., 2016; Stromer \& Osborne, 1982), encobrir um deles com uma máscara (McIlvane et al., 1987; McIlvane et al., 1984), ou os próprios testes de equivalência (Carrigan \& Sidman, 1992; Johnson \& Sidman, 1993; Perez et al., 2015). Embora esses testes possam fornecer uma medida do controle prevalente ao longo das tentativas de treino (medida intertentativas), não permitem que seja verificado o estabelecimento dos controles por seleção ou rejeição tentativa-a-tentativa (medida intratentativa). A possibilidade de se ter uma medida desses controles em cada uma das tentativas de treino, e não somente em testes posteriores, aumentaria a confiabilidade dos dados, bem como permitiria verificar em que medida os testes realizados de fato oferecem informações fidedignas acerca dos controles estabelecidos durante o treino.

Estudos analítico-comportamentais utilizaram os movimentos dos olhos como uma medida auxiliar em investigações sobre controle de estímulos (e.g., Dube et al., 2006; 2010; Endemann, et al., 2011; Huziwara et al., 2015, 2016; Perez et al., 2015; Pessôa et al., 2009). Magnusson (2002) utilizou um rastreador de movimentos dos olhos para verificar como a ocorrência dos controles por seleção e por rejeição afetaria a observação (fixação) dos estímulos de comparação. Foi avaliada, em especial, a ocorrência de tentativas em que os participantes escolhiam um dos estímulos de comparação após observação exclusiva do S+ ou do S- (i.e., a estratégia ou topografia da observação). Dois participantes foram expostos a duas condições experimentais, ambas compostas por treinos de relações condicionais seguidos de testes de equivalência. Em uma das condições, o controle por rejeição era favorecido para o treino das relações condicionais entre os conjuntos de estímulos $\mathrm{AB}$ e BC (condição RJ); em outra condição, o controle por seleção era favorecido para o treino das relações condicionais entre os conjuntos DE e EF (condição SL). Para favorecer o estabelecimento de controle por rejeição (para uma revisão, ver Perez \& Tomanari, 2014), durante o treino, cada modelo era sistematicamente apresentado simultaneamente a um mesmo S- (A1 e B2), ao passo que o $\mathrm{S}+$ variava a cada tentativa $(\mathrm{B} 1, \mathrm{X} 1, \mathrm{X} 2, \mathrm{X} 3 \ldots)$. Além disso, um procedimento de dica atrasada foi utilizado para evitar o controle pelo S+. Depois de apresentados os estímulos na tela (e.g., A1-B1B2), transcorrido um dado intervalo na ausência de respostas do participante, o estímulo de comparação $\mathrm{S}+$ (B1) era substituído por um quadrado branco e somente o modelo e o S- permaneciam disponíveis para observação. Respostas de escolha do quadrado branco eram, a partir de então, seguidas das consequências programadas para acerto. Inicialmente, tal dica era apresentada $0,1 \mathrm{~s}$ depois da apresentação dos estímulos de comparação. $\mathrm{O}$ período necessário à apresentação da dica atrasada foi 
progressivamente aumentado com o decorrer do treino, até se tornar dispensável. $\mathrm{Na}$ condição $\mathrm{SL}$, para favorecer o estabelecimento do controle por seleção, cada modelo era apresentado simultaneamente a um mesmo S+ (A1 e B1), ao passo que o $\mathrm{S}$ - variava a cada tentativa (B2, Y1, Y2, Y3...). Além disso, depois de apresentados os estímulos na tela (A1-B1B2), o S- era apagado sob vigência do procedimento de dica atrasada. Durante a condição RJ, um dos participantes apresentou baixos escores nos testes de reflexividade (AA, BB e $\mathrm{CC}$ ), transitividade ( $\mathrm{AC}$ ) e equivalência (CA), e altos escores nos testes de simetria, indicando, assim, o estabelecimento do controle por rejeição (ver Carrigan \& Sidman, 1992). O outro participante apresentou um padrão similar, porém não tão claramente, sendo necessária a repetição dos testes. $\mathrm{Na}$ condição SL, os participantes apresentaram alta porcentagem de acerto em todos os testes, indicando o estabelecimento do controle por seleção. Para um dos participantes, na condição RJ, observou-se uma alta frequência de escolhas realizadas após fixar somente o S-; o inverso se deu na condição SL, ou seja, houve uma alta frequência das escolhas realizadas após fixar somente o $\mathrm{S}+$. Para outro participante, não houve diferenças na estratégia de observação dos estímulos em função das condições experimentais.

O estudo de Magnusson (2002), no entanto, apresenta limitações. Primeiramente, o uso da dica atrasada durante o treino das relações condicionais pode ter afetado o padrão de observação dos estímulos. No procedimento de dica atrasada, um dos estímulos de comparação é retirado da tela após a passagem de um breve intervalo. Necessariamente, tal procedimento afeta o padrão de observação dos estímulos de comparação, uma vez que somente um deles fica disponível na tela, podendo ser observado com maior frequência e duração comparativamente ao outro. Assim, tal procedimento impede uma comparação da observação de S+ vs. S-. Portanto, para se investigar a observação de $\mathrm{S}+$ e S- durante o treino das relações condicionais e seus efeitos sobre o estabelecimento dos controles por seleção ou por rejeição, seria importante a manutenção de ambos os estímulos de comparação na tela, ao longo de todas as tentativas de treino. Para além da possível interferência do procedimento de dica atrasada, a medida escolhida por Magnusson (2002), no caso, a estratégia de observação de $\mathrm{S}+$ ou S- antes de realizar a escolha, também pode ter mascarado diferenças importantes na observação dos estímulos. Por exemplo, embora um dos participantes não tenha apresentado diferenças entre as condições experimentais com relação à observação exclusiva de $\mathrm{S}+$ ou S-, é possível que tenha havido diferenças na frequência ou duração relativas da observação de $\mathrm{S}+$ e $\mathrm{S}-$, como já documentado em outros estudos (e.g., (Dube et al., 2006; 2010; Huziwara et al., 2016; Perez et al., 2015; Pessôa et al., 2009; Steingrimsdottir \& Arnsten, 2016).

Huziwara et al. (2016), por exemplo, investigaram a relação entre a duração total da observação dos estímulos em treinos de discriminação condicional e sua relação com a ocorrência dos controles por seleção ou por rejeição. Para tanto, expuseram os participantes a treinos de discriminação condicional seguidos por testes de equivalência e por sondas, com estímulos novos, para checar o estabelecimento dos controles por seleção ou por rejeição. Os resultados dos testes de equivalência e das sondas indicaram que ambos os controles foram estabelecidos para todos os participantes. Sob essas condições, Huziwara et al. avaliaram a duração total da observação de $\mathrm{S}+$ e $\mathrm{S}-$. Os resultados mostraram que, depois do modelo, o estímulo observado com maior duração foi o $\mathrm{S}+$, seguido do S-. O estudo de Huziwara et al., no entanto, apontou dificuldades que impediram conclusões sobre a relação entre a observação dos estímulos de comparação e o estabelecimento dos controles por seleção ou por rejeição. Como ambos controles foram verificados na mesma condição de treino, não foi possível encontrar diferenças na observação relacionadas a cada um desses controles, separadamente. Para tanto, como em Magnusson (2002), os controles por seleção e por rejeição deveriam ter sido estabelecidos separadamente, em condições de treino distintas, para diferentes conjuntos de estímulos. Além disso, também foi observado um efeito da prática, ou seja, uma redução da observação dos estímulos ao longo do experimento (ver também Pessôa et al., 2009). Como reportado em outros estudos, tal problema poderia ser minimizado com a redução do tamanho dos estímulos (Perez et al., 2015; Steingrimsdottir \& Arnsten, 2016) ou com o igualação da luminância (Pessôa et al., 2009).

O presente estudo teve por objetivo investigar a relação entre a observação dos estímulos de comparação com função de $\mathrm{S}+$ e S- durante o treino de relações condicionais e a ocorrência dos controles por seleção ou por rejeição medida pelos testes de equivalência. Para tanto, foi realizada uma replicação sistemática do estudo de Magnusson (2002), buscando enviesar o estabelecimento dos controles por rejeição ou por seleção, e acrescentando uma condição experimental de linha de base, na qual nenhum controle específico foi favorecido. A programação dessas três condições distintas teve por objetivo permitir uma comparação mais clara das diferenças na observação em função das condições manipuladas nos treinos das relações condicionais. Além disso, pretendeu-se avaliar não somente as estratégias de observação antes da escolha do estímulo de comparação (cf. Magnusson, 2002), mas também a frequência e a duração relativa da observação dos estímulos S+ e S- (cf., Huziwara et al., 2016; ver também Perez et al., 2015). De modo a evitar qualquer perturbação da medida de frequência e duração relativa durante o treino, diferentemente de Magnusson, o procedimento de dica atrasada não foi utilizado no presente estudo. Ainda, seguindo a indicação de Huziwara et al. (2016), o tamanho dos estímulos foi reduzido de modo a minimizar o efeito da prática e a perda da medida da fixação dos estímulos. 


\section{MÉTODO}

\section{Participantes}

Participaram do experimento quatro estudantes universitários, com idade entre 18 e 25 anos, sem histórico de atuação em pesquisas em Psicologia. Antes da realização do experimento, os participantes leram e assinaram o "Termo de Consentimento Livre e Esclarecido" (previamente aprovado pelo Comitê de Ética em Pesquisa com Seres Humanos do IP-USP, Prot.0807/CEPH-IP/23/04/2007).

\section{Local, Equipamentos e Estímulos}

A pesquisa foi realizada em uma sala com 2,0 $\mathrm{m} \times 3,5$ $\mathrm{m}$, dividida por um biombo. De um lado do biombo havia uma cadeira e uma mesa sobre a qual estavam dispostos um rastreador de movimentos dos olhos (ISCAN ${ }^{\circledR}, 2004$, Corp., Burlington, MA; www.iscaninc.com), uma tela de computador de 17 polegadas, duas caixas de som padrão e um teclado que foi utilizado pelos participantes. Do outro lado, foram dispostos os equipamentos utilizados pelo experimentador, descritos abaixo. Uma descrição completa do equipamento e suas rotinas de calibragem pode ser encontrado em outras publicações (e.g., Endemann et al., 2011; Huziwara et al., 2015, 2016; Perez et al., 2015)

O software MTS 11.6.7 (Dube \& Hiris, 1999), executado em um computador Macintosh Performa 5215CD, foi utilizado para controlar, durante as sessões experimentais, a apresentação dos estímulos no monitor do participante, o registro das respostas dadas no teclado e a apresentação de consequências.

Em todas as condições experimentais, estímulos visuais de aproximadamente $0,5 \mathrm{~cm}$ x $0,5 \mathrm{~cm}$ foram utilizados como modelo e comparações. A uma distância de aproximadamente $60 \mathrm{~cm}$ entre o olho do participante e a tela, os estímulos teriam medidas angulares de aproximadamente $0,5^{\circ} \times 0,5^{\circ}$. No Pré-treino e na condição de Linha de Base (ver adiante) foram utilizadas figuras sem sentido do banco de figuras do MTS 11.6.7; nas condições de controle por Rejeição (RJ) e controle por Seleção (ver adiante) foram utilizadas letras do alfabeto ocidental em fonte Times New Roman e letras do alfabeto grego, à semelhança dos estímulos utilizados por Magnusson (2002).

As figuras poderiam ser apresentadas em cinco áreas de fundo branco, medindo $2,5 \mathrm{~cm} \mathrm{x} 2,5 \mathrm{~cm}\left(2,4^{\circ} \times 2,4^{\circ}\right)$, localizadas nos quatro cantos e no centro da tela (metade do tamanho utilizado por Huziwara et al., 2016). A distância entre os pontos centrais das áreas dos cantos superiores e entre as áreas dos cantos inferiores era de $31 \mathrm{~cm}\left(29^{\circ}\right)$; a distância entre as áreas superiores e inferiores era de 23 $\mathrm{cm}\left(21,7^{\circ}\right)$; e a distância entre as áreas dos cantos da tela e a área central era de $19,5 \mathrm{~cm}\left(18,5^{\circ}\right)$. O fundo da tela era preto.

\section{Procedimento}

O procedimento foi iniciado com um pré-treino do uso do teclado para a realização da tarefa. Na sequência, o participante foi exposto a três diferentes condições experimentais: Linha de Base (LB), Controle por Rejeição (RJ) e Controle por Seleção (SL). Dois dos participantes (P1 e P2) foram expostos à seguinte sequência de condições: LB $\rightarrow \mathrm{RJ} \rightarrow \mathrm{SL}$; os outros dois (P3 e P4) tiveram a ordem das duas últimas condições invertidas ( $\mathrm{LB} \rightarrow \mathrm{SL} \rightarrow \mathrm{RJ}$ ). A cada dia que o participante comparecia ao laboratório, uma das três condições experimentais era realizada do início ao fim da sessão experimental. O tempo diário necessário à calibragem do ISCAN ${ }^{\circledR}$ e à coleta de dados variou de 40 a 75 minutos.

Todas as condições experimentais eram compostas por blocos de treino de discriminação condicional e de teste das propriedades definidoras de uma de classes de estímulos equivalentes. Em ambos os casos, um estímulo modelo era apresentado no centro da tela. Diante do modelo, o participante deveria apertar a tecla "K". Tal resposta era seguida pela apresentação de dois estímulos de comparação nos cantos da tela. Para escolher o estímulo de comparação do canto inferior esquerdo, o participante deveria apertar a tecla "J"; para escolher o estímulo do canto inferior direito, a tecla "L"; para o estímulo do canto superior esquerdo, a tecla "U"; e para o estímulo do canto superior direito, a tecla "O". Depois de escolher um dos estímulos de comparação, todos os estímulos apresentados naquela tentativa desapareciam da tela. Nas sessões de treino, respostas aos estímulos arbitrariamente designados como "corretos" $(\mathrm{S}+)$ foram classificadas como "acertos" e eram seguidas do acúmulo de um ponto em um contador localizado no centro superior da tela, medindo $1,5 \mathrm{~cm} \mathrm{x} 1 \mathrm{~cm}\left(1,4^{\circ} \times 1^{\circ}\right)$, bem como de um som de "bip". Respostas aos estímulos arbitrariamente designados como "incorretos" (S-) foram classificadas como "erros" e eram seguidas de um ruído "bzzz". As tentativas foram separadas por um intervalo entre tentativas (IET) de $0,5 \mathrm{~s}$. Nas sessões de treino em extinção e nas sessões de teste, não foram apresentadas consequências programadas para acerto e erro. Nessas sessões, a resposta de escolha de um dos estímulos de comparação era seguida do IET e do início de uma nova tentativa.

O balanceamento das tentativas se deu de tal forma que, durante uma sessão: (a) os estímulos modelo deveriam ser apresentados um mesmo número de vezes; (b) o mesmo estímulo modelo não poderia ser apresentado por mais do que três tentativas consecutivas; (c) os estímulos de comparação deveriam ser apresentados o mesmo número de vezes em cada canto da tela; e (d) o mesmo estímulo comparação não poderia ser apresentado na mesma posição por mais do que três tentativas consecutivas.

Linha de Base (LB). Nessa condição, uma mesma proporção de $\mathrm{S}+/ \mathrm{S}$ - foi utilizada ao longo do treino das 
relações condicionais. Assim, nenhuma relação de controle modelo/comparação específica foi favorecida. $\mathrm{O}$ treino relacional e os testes foram realizados tal como descrito acima.

Foram utilizados três conjuntos de estímulos (A, B e C) compostos por dois estímulos cada (A1 e A2; B1 e B2; e C1 e $\mathrm{C} 2$, respectivamente). Primeiramente, foram ensinadas as relações AB. Os estímulos do conjunto A (A1 e A2) foram apresentados sucessivamente como modelos; os estímulos do conjunto B (B1 e B2) foram apresentados simultaneamente como comparações. Nesta e em todas as outras condições do experimento, cada bloco de tentativas era formado por quatro tentativas de cada uma das relações treinadas, de modo a garantir que cada estímulo de comparação aparecesse uma vez em cada um dos quatro cantos da tela, procurando evitar, assim, eventuais controles por posição. Dessa forma, cada sessão de treino era formada por 16 tentativas apresentadas em dois blocos de oito tentativas cada, sendo quatro tentativas A1-B1B2 e quatro tentativas A2-B1ㅡㄹ (o estímulo de comparação sublinhado é o $\mathrm{S}+$ ). Para que o treino $\mathrm{AB}$ fosse finalizado, o participante deveria apresentar não mais do que um único erro durante a sessão, o que equivale a $94 \%$ de acertos. Esse critério de aprendizagem foi utilizado em todas as fases de treino seguintes.

$\mathrm{Na}$ sequência, foram ensinadas a relações $\mathrm{BC}$. Os estímulos do conjunto B (B1 e B2) foram apresentados sucessivamente como modelos; os estímulos do conjunto $\mathrm{C}$ ( $\mathrm{C} 1$ e $\mathrm{C} 2)$ foram apresentados simultaneamente como comparações. As sessões de treino $\mathrm{BC}$ foram programadas utilizando os mesmos critérios das sessões de treino $A B$, porém com as seguintes configurações de tentativa: $\mathrm{B} 1$ $\underline{\mathrm{C} 1 \mathrm{C} 2}$ e $\mathrm{B} 2-\mathrm{C} 1 \underline{\mathrm{C}} 2$.

Uma vez ensinadas separadamente as relações $A B$ e $\mathrm{BC}$, foi realizado o treino misto $\mathrm{AB} / \mathrm{BC}$. Para tanto, cada sessão de treino foi formada por 32 tentativas divididas em dois blocos de 16 tentativas, sendo quatro para cada uma das configurações "modelo-comparações" apresentadas. O critério de encerramento da sessão era o mesmo do treino $\mathrm{AB}$ (não mais do que um erro).

Antes do início dos testes, foi apresentada ao participante a seguinte instrução: "Agora, o computador não mais lhe dirá se sua escolha foi correta ou incorreta. Faça o melhor que puder". O participante era, então, exposto a um bloco de tentativas de treino $\mathrm{AB} / \mathrm{BC}$. Caso o responder se mantivesse em acordo com o critério de aprendizagem, os testes de equivalência eram iniciados.

Em seguida, foram testadas, sequencialmente, as seguintes relações: transitividade (AC), simetria (BA e $\mathrm{CB})$, equivalência $(\mathrm{CA})$ e reflexividade (AA, BB e CC). Foi realizada uma sessão de teste para cada uma das relações testadas. Cada sessão de teste era composta por 32 tentativas: 16 tentativas de treino (um bloco misto de 16 tentativas $\mathrm{AB} / \mathrm{BC}$ ) e 16 tentativas de teste, oito para cada uma das configurações "modelo-comparações" específicas de cada teste (e.g., $\mathrm{A} 1-\underline{\mathrm{C} 1} \mathrm{C} 2$ e $\mathrm{A} 2-\mathrm{C} 1 \underline{\mathrm{C} 2}$, no teste $\mathrm{AC}$; $\mathrm{C} 1-\underline{\mathrm{B} 1 \mathrm{~B} 2}$

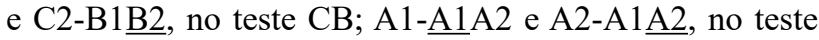
AA etc.) Em todas as sessões de teste, desta e das demais condições (RJ e SL), as tentativas de treino e de teste não foram seguidas de consequências diferenciais programadas para acerto e erro.

Controle por Rejeição (RJ). Nessa condição, tevese por objetivo favorecer o estabelecimento de relações modelo/S-. Primeiramente, foram ensinadas as relações DE. Os estímulos do conjunto D (D1 e D2) foram apresentados sucessivamente como modelos, seguidos da apresentação de dois estímulos, um do conjunto E (E1 e E2) e outro do conjunto X (X1, X2, X3, X4, X5 e X6), apresentados simultaneamente como comparações. Com o objetivo de favorecer o estabelecimento do controle por rejeição, o S- foi apresentado sucessivamente junto a quatro outros estímulos de comparaçâo com função de S+ (Carrigan \& Sidman, 1992; Johnson \& Sidman, 1993; Magnusson, 2002; Perez et al., 2015). Quando o modelo era D1, E2 (S-) era sucessivamente apresentado junto a E1, X1, X2 e X3 (S+) como comparações (D1-E1E2, D1-X1E2, D1-X2E2, D1$\underline{\mathrm{X}} \mathrm{E} 2$ ); quando o modelo era D2, E1 (S-) era sucessivamente apresentado junto a E2, X4, X5 e X6 (S+) (D2-E1E2, D2-E1 $\underline{X 4}, \mathrm{D} 2-\mathrm{E} 1 \underline{\mathrm{X}}$, D2-E1 $\underline{\mathrm{X}})$. Cada sessão de treino foi formada por 64 tentativas divididas em dois blocos de 32 tentativas, sendo quatro para cada uma das configurações "modelo-comparações". O critério de encerramento do treino foi o mesmo utilizado na condição de LB.

$\mathrm{Na}$ sequência, foram treinadas as relações EF. De forma análoga ao treino DE, os estímulos do conjunto E (E1 e E2) foram apresentados sucessivamente como modelos seguidos da apresentação de dois estímulos, um do conjunto $\mathrm{F}$ (F1 e F2) e outro do conjunto Y (Y1, Y2, Y3, Y4, Y5 e Y6), apresentados simultaneamente como comparações. Quando o modelo era E1, F2 (S-) era sucessivamente apresentado junto a F1, Y1, Y2 e Y3 (S+) como comparações (E1-F1F2, E1-Y1F2, E1-Y2F2, E1-Y3F2); quanto o modelo era E2, F1 (S-) era sucessivamente apresentado junto a F2, Y4,

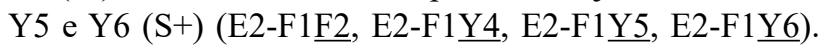
As sessões de treino EF foram programadas utilizando os mesmos critérios das sessões de treino DE.

Uma vez treinadas separadamente as relações DE e EF, realizou-se ainda um treino misto DE/EF. Para tanto, foi realizado um treino com 128 tentativas divididas em dois blocos de 64 tentativas, quatro para cada uma das configurações "modelo-comparações". Os testes dessa condição foram realizados de forma idêntica aos testes da condição LB e só foram iniciados depois de o participante apresentar pelo menos $97 \%$ de acerto em uma sessão de treino $\mathrm{DE} / \mathrm{EF}$ sem consequências programadas para acerto e erro.

Foram testadas as seguintes relações, na ordem apresentada: transitividade (DF), simetria (ED e FE), simetria da transitividade (FD) e reflexividade (DD, EE e FF). Foi realizada uma sessão de teste para cada uma das relações testadas. Cada sessão de teste era composta por 80 tentativas: 64 tentativas de treino (um bloco misto de 64 
tentativas $\mathrm{DE} / \mathrm{EF}$ ) e 16 tentativas de teste, oito para cada uma das configurações "modelo-comparações" (e.g., D1-F1F2 e D2-F1느, no teste DF; F1-E1E2 e F2-E1ㄹ2, no teste FE; D1- $\underline{D} 1 \mathrm{D} 2$ e D2-D12, no teste DD etc.)

Controle por Seleção (SL). Nessa condição, o objetivo era favorecer o estabelecimento de relações modelo/S+. Em um primeiro momento, foram ensinadas as relações $\mathrm{GH}$. Os estímulos do conjunto $\mathrm{G}$ (G1 e G2) foram apresentados sucessivamente como modelos, seguidos da apresentação de dois estímulos, um do conjunto $\mathrm{H}(\mathrm{H} 1$ e $\mathrm{H} 2)$ e outro do conjunto $\mathrm{Z}$ (Z1, Z2, Z3, Z4, Z5 e Z6), apresentados simultaneamente como comparações. Com o objetivo de favorecer o estabelecimento de relação modelo/S+, o $\mathrm{S}+$ foi apresentado sucessivamente junto a quatro outros comparações com função de S- (Carrigan \& Sidman, 1992; Magnusson, 2002; Perez et al., 2015). Quando o modelo era G1, H1 (S+) era sucessivamente apresentado junto a H2, Z1,

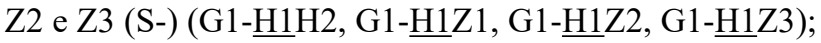
quanto o modelo era $\mathrm{G} 2, \mathrm{H} 2(\mathrm{~S}+)$ era sucessivamente apresentado junto a H1, Z4, Z5 e Z6 (S-) (G2-H1늘, G2-Z4는, G2-Z5 $\underline{\mathrm{H} 2}, \mathrm{G} 2-\mathrm{Z} 6 \underline{\mathrm{H} 2}$ ). Cada sessão de treino foi formada por 64 tentativas divididas em dois blocos de 32 tentativas, sendo quatro para cada uma das configurações. $\mathrm{O}$ critério de encerramento dos passos de treino foi o mesmo utilizado nas condições anteriores.

Em seguida, foram treinadas as relações HI. De forma análoga ao treino $\mathrm{GH}$, os estímulos do conjunto $\mathrm{H}$ (H1 e H2) foram apresentados sucessivamente como modelos seguidos da apresentação de dois estímulos, um do conjunto I (I1 e I2) e um do conjunto W (W1, W2, W3, W4, W5 e W6), apresentados simultaneamente como comparações. Quando o modelo era $\mathrm{H} 1, \mathrm{I} 1(\mathrm{~S}+)$ era sucessivamente apresentado junto de I2, W1, W2 e W3 (S-) (H1-I1I2, H1-I1W1, H1I1W2, H1-I1W3); quanto o modelo era H2, I2 (S+) era sucessivamente apresentado junto a I1, W4, W5 e W6 (S-)

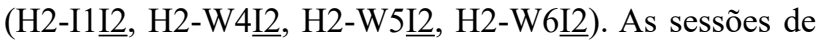
treino $\mathrm{HI}$ foram programadas utilizando os mesmos critérios das sessões de treino GH.

Uma vez treinadas separadamente as relações GH e HI, realizou-se ainda um treino misto GH/HI. Para tanto, foi realizado um treino com 128 tentativas divididas em dois blocos de 64 tentativas, quatro para cada tipo de tentativa. Os testes dessa condição foram realizados de forma idêntica aos testes das condições anteriores (LB e RJ). Foram testadas as seguintes relações na ordem apresentada: transitividade (GI), simetria (HG e IH), simetria da transitividade (IG) e reflexividade (GG, HH e II). Foi realizada uma sessão de teste para cada uma das relações testadas. Cada sessão de teste era composta por 80 tentativas: 64 tentativas de treino (um bloco misto de 64 tentativas GH HI) e 16 tentativas de teste, oito para cada uma das configurações "modelocomparações" (e.g., G1-I112 e I2-I1 $\underline{\text { I2}}$, no teste GI; H1-G1G2 e H2-G1G2, no teste HG; H1- $\underline{\mathrm{H} 1 \mathrm{H} 2}$ e $\mathrm{H} 2-\mathrm{H} 1 \underline{\mathrm{H} 2}$, no teste HH etc.)

\section{RESULTADOS}

Ao longo do treino das condições experimentais, de modo geral, os participantes levaram de três a 11 sessões para atingir o critério de aprendizagem para todas as relações condicionais. $\mathrm{O}$ participante $\mathrm{P} 3$ foi a exceção e precisou de 29 sessões para atingir o critério na condição LB. Para todos os participantes, o número de sessões de treino na condição LB foi maior do que nas condições seguintes e o número de sessões de treino na condição SL foi maior do que na condição RJ. Na sessão de treino sem consequências programadas, que precedeu a realização dos testes de cada condição, todos os participantes mantiveram o desempenho similar ao da última sessão de treino com feedback.

Durante os testes das condições LB e SL, em geral, todos os participantes apresentaram resultados condizentes com a formação de classes de estímulos equivalentes, ou seja, apresentaram altas porcentagens de acerto (pelo menos 14 em 16 tentativas) nos testes de transitividade, simetria, equivalência e reflexividade. A única exceção foi o participante $\mathrm{P} 2$, no teste CA $(81 \%, 13$ acertos em 16 tentativas). Tais resultados, tomados com um todo, indicam o estabelecimento do controle por seleção.

Já na condição RJ, apenas a participante $\mathrm{P} 1$ apresentou resultados que indicam o estabelecimento do controle por rejeição, ou seja, falhas sistemáticas nos testes de transitividade, equivalência e reflexividade. A participante apresentou um único acerto $(6 \%)$ nas sessões de DF (transitividade), FD (equivalência) e EE (reflexividade). Nas sessões DD e FF (reflexividade), a participante não emitiu qualquer resposta de escolha correta. Já nos testes de simetria (ED e FE), apresentou alta porcentagem de acerto. Os demais participantes, P2, P3 e P4, de maneira geral, apresentaram alta porcentagem de acerto em todos os testes, o que indica controle por seleção; a única exceção foi o participante P4, no teste DF ( $0 \%$ de acerto), de acordo com a Tabela 1.

Considerando que o presente experimento utilizou procedimentos para favorecer o estabelecimento dos controles por seleção ou por rejeição, para a análise dos movimentos dos olhos foi considerada somente a última sessão de treino de cada condição experimental. Durante as condições de treino, os padrões de observação apresentaram elevada variabilidade e, por essa razão, deu-se preferência para a avaliação da duração e da frequência de fixação aos estímulos em condições já estáveis de responder discriminado. A duração de uma fixação corresponde ao tempo decorrido entre a entrada e a saída do cursor gerado pelo ISCAN ${ }^{\circledR}$ na área de um estímulo. A frequência de fixações, por sua vez, corresponde ao número de entradas do cursor em uma dada área. Uma sessão de cada 
Tabela 1

Frequência de ocorrência das estratégias de observar somente um dos estímulos de comparação (somente $S+$ ou somente $S$-) antes de emitir a resposta de escolha, na última sessão de treino das condições experimentais $L B, R J$ e $S L$.

\begin{tabular}{|c|c|c|c|}
\hline \multirow{2}{*}{ Participantes } & \multirow{2}{*}{ Condições } & \multicolumn{2}{|c|}{ Frequência } \\
\hline & & Observar somente S+ & Observar somente S- \\
\hline \multirow{3}{*}{ P1 } & LB & 5 & 0 \\
\hline & RJ & 18 & 30 \\
\hline & SL & 33 & 7 \\
\hline \multirow{3}{*}{$\mathrm{P} 2$} & LB & 9 & 5 \\
\hline & RJ & 26 & 10 \\
\hline & SL & 43 & 4 \\
\hline \multirow{3}{*}{ P3 } & LB & 2 & 1 \\
\hline & $\mathrm{RJ}$ & 0 & 4 \\
\hline & SL & 2 & 7 \\
\hline \multirow{3}{*}{$\mathrm{P} 4$} & LB & 4 & 0 \\
\hline & RJ & 0 & 4 \\
\hline & SL & 14 & 0 \\
\hline
\end{tabular}

participante foi avaliada por um observador independente. A concordância entre observadores foi calculada dividindo-se o número de acordos pelo número de acordos somado ao de desacordos. A média do acordo entre observadores ao longo das sessões analisadas foi de $94 \%$.

A Figura 1 apresenta a duração e a frequência relativa das observações para $\mathrm{S}+$ e $\mathrm{S}$ - na última sessão de treino de cada condição experimental. Tal resultado foi obtido dividindo-se os valores absolutos da duração e da frequência da observação de S+ pelos valores da observação de S- ao longo de toda a condição. Assim, pontos acima da linha (valor 1) correspondem a uma maior duração ou frequência da observação para $\mathrm{S}+$, enquanto que pontos abaixo da linha correspondem a uma maior duração ou frequência da observação para S-. Assim, daqui em diante, a descrição de uma maior ou menor observação de $\mathrm{S}+$ ou S- será sempre baseada na comparação relativa entre a observação total de ambos os estímulos em uma dada condição experimental.

A Figura 1 mostra que, para a maioria dos participantes, tanto a duração quanto a frequência relativa da observação do $\mathrm{S}+$ foi maior em todas as condições do procedimento. A única exceção ocorreu para a participante $\mathrm{P} 1$, durante a condição RJ, em que tanto a duração quanto a frequência relativa da observação foi maior para S-. Para os demais participantes (P2, P3 e P4), com relação à duração da observação, observa-se que a introdução da condição RJ foi acompanhada de uma redução na duração da observação relativa de $\mathrm{S}+$. Para esses mesmos participantes, a mudança para a condição SL foi acompanhada do aumento na duração da observação relativa de $\mathrm{S}+$. Os resultados da frequência relativa de observação dos estímulos de comparação, por outro lado, foram mais variáveis ao longo das condições experimentais RJ e SL. Para o participante P2 a frequência relativa de observação dos comparações só foi alterada na condição SL, na qual o $\mathrm{S}+$ foi o estímulo de comparação mais observado; no caso do participante P3, houve uma maior frequência de observação de $\mathrm{S}+$ na condição $\mathrm{LB}$, seguido de uma maior frequência de observação de S- na fase RJ e uma igualação da frequência relativa de observação dos estímulos de comparação na condição SL. O participante P4, por fim, apresentou uma redução progressiva na frequência de observação de $\mathrm{S}+$ ao longo das condições experimentais. A única regularidade observada para os quatro participantes foi a maior frequência de observação de $\mathrm{S}+$ na condição LB.

Duas estratégias de observação foram consideradas como indicativos das relações de controle investigadas. $\mathrm{O}$ registro de uma resposta de escolha após a observação exclusiva do modelo e do S- foi considerada como indicativo de controle por rejeição; por outro lado, o registro de uma escolha após a observação exclusiva do modelo e do $\mathrm{S}+$ foi considerada como indicativo de controle por seleção. A Tabela 1 apresenta a frequência dessas duas estratégias durante a última sessão de treino de cada condição experimental. É possível verificar que, para os participantes P1, P2 e P4, na condição LB, escolhas baseadas na observação exclusiva de $\mathrm{S}+$ foram mais frequentes do que escolhas baseadas na observação exclusiva de S-. De maneira especial para a participante $\mathrm{P} 1$, que apresentou resultados indicativos de controle por rejeição nos testes de equivalência durante a condição RJ e resultados indicativos de controle por seleção nos testes da condição SL, a comparação da estratégia mais frequente em cada condição experimental revela um padrão consistente entre a observação e o estabelecimento de cada tipo de controle. Para a participante P1, é possível verificar uma maior frequência de escolhas realizadas após observação somente de S- na condição RJ e uma maior frequência de escolhas após observação exclusiva de $\mathrm{S}+$ na 

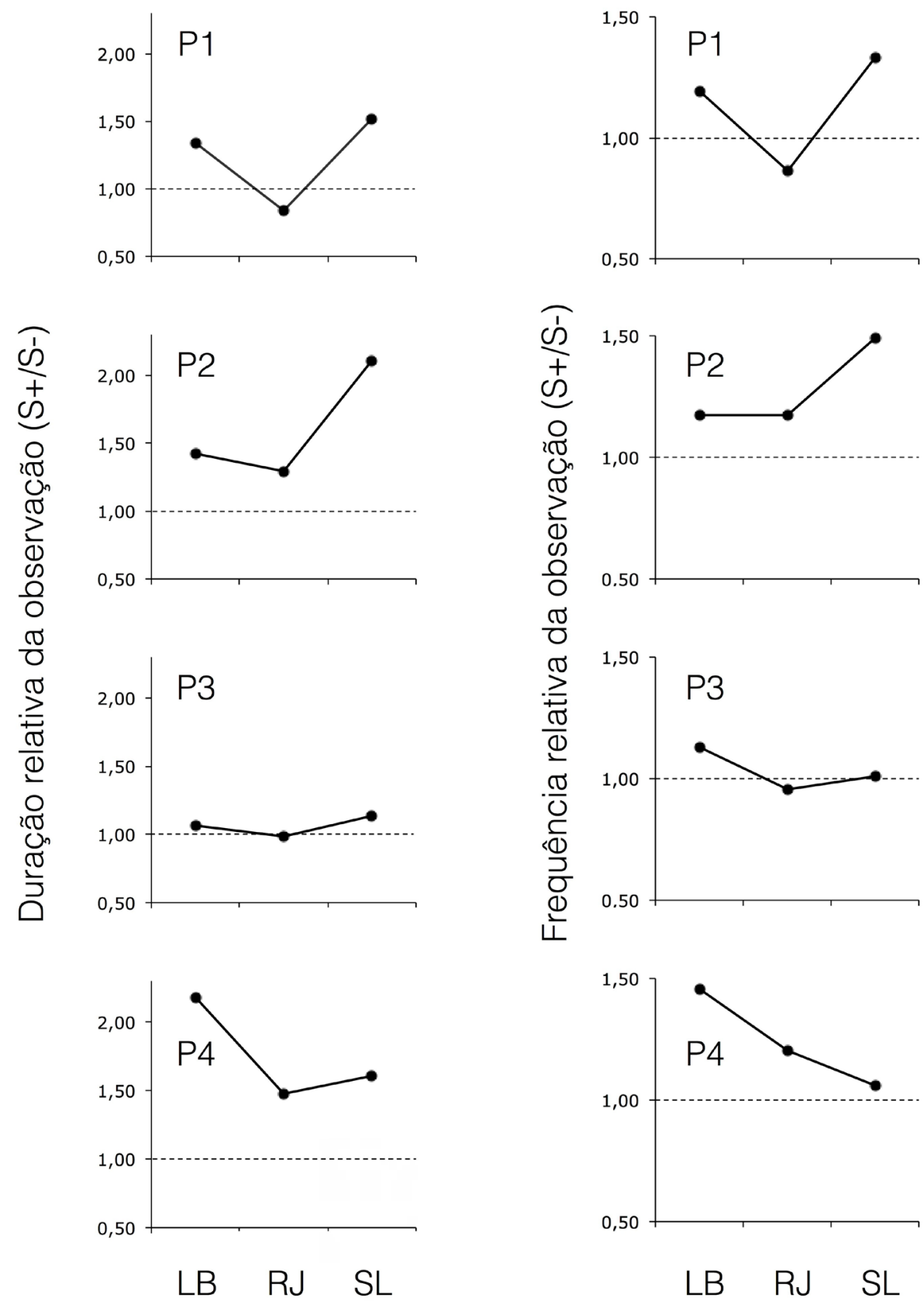

\section{Condições experimentais}

Figura 1. Duração (painéis à esquerda) e frequência (painéis à direita) relativa da observação de $\mathrm{S}+$ durante a última sessão de treino de cada condição experimental (LB, RJ e SL) para cada participante.

condição SL. Um padrão similar pode ser observado para o participante $\mathrm{P} 4$, embora em menor frequência. Ainda comparando as condições RJ e SL, para o participante P2, a observação exclusiva de $\mathrm{S}+$ foi mais frequente a despeito da condição; para o participante $\mathrm{P}$, a frequência de respostas de escolha após a observação exclusiva de apenas um dos estímulos de comparação foi pouco frequente, com uma tendência maior à observação exclusiva de S-. 


\section{DISCUSSÃO}

O presente experimento teve por objetivo avaliar o efeito dos controles por seleção e por rejeição sobre a frequência e duração relativa da observação de $\mathrm{S}+$, bem como sobre as estratégias de observação (observação exclusiva do $\mathrm{S}+$ ou do S-) durante uma tarefa de emparelhamento com o modelo (MTS), avaliadas por meio dos movimentos dos olhos. Os participantes foram expostos a três condições experimentais que envolveram, para diferentes conjuntos de estímulo, treinos de discriminação condicional seguidos de testes de equivalência. Na condição LB, nenhum controle específico foi favorecido; nas condições RJ e SL, foi utilizado um procedimento para favorecer o estabelecimento dos controles por rejeição e por seleção, respectivamente. $\mathrm{Na}$ condição LB, todos os participantes apresentaram resultados positivos nos testes de equivalência; em geral, a frequência e a duração da observação dos estímulos de comparação foi relativamente maior para $\mathrm{S}+$; além disso, escolhas realizadas após a observação exclusiva do $\mathrm{S}+$ foram mais frequentes do que após observação exclusiva do S-. Na condição RJ, considerando os testes de equivalência, apenas um participante (P1) apresentou resultados que atestaram o estabelecimento do controle por rejeição. Para esse participante, a duração e a frequência da observação dos estímulos de comparação foi maior para o S- do que para o $\mathrm{S}+$; também foram mais frequentes escolhas realizadas após a observação exclusiva do S-. Para os demais participantes, a condição RJ foi acompanhada de uma redução da observação relativa de $\mathrm{S}+$, comparativamente às demais fases. $\mathrm{Na}$ condição $\mathrm{SL}$, os resultados dos quatro participantes indicaram o estabelecimento do controle por seleção; para três participantes (P1, P2 e P4), foi observada uma maior duração relativa de observação de $\mathrm{S}+\mathrm{e}$, para dois participantes $(\mathrm{P} 1 \mathrm{e}$ P2), também uma maior frequência relativa da observação de $\mathrm{S}+$ nessa condição. Ainda, para três dos quatro participantes (P1, P2 e P4), foi verificado uma maior ocorrência de escolhas após observação exclusiva do $\mathrm{S}+$.

Durante os testes das condições LB e SL, todos os participantes apresentaram resultados condizentes com a formação de classes de estímulos equivalentes, ou seja, apresentaram altas porcentagens de acerto (próximas ou iguais a 100\%) nos testes de transitividade, simetria, equivalência e reflexividade. Na condição RJ, os participantes P2, P3 e P4, de modo geral, também apresentaram resultados positivos em todos os testes. A participante P1, na condição RJ, ao contrário dos demais participantes, apresentou falhas sistemáticas nos testes de transitividade, equivalência e reflexividade - ou seja, apresentou uma porcentagem de acerto próxima ou igual a zero, um desempenho típico de rejeição, como previsto por Carrigan e Sidman (1992, 2020; ver também Johnson \& Sidman, 1993; Perez et al., 2015, 2017, 2020).

A pronta emergência das relações condicionais sob controle por rejeição, ou seja, escores próximos a zero nos testes de transitividade, equivalência e reflexividade e escores elevados nos testes de simetria, tal como observado nos resultados da participante $\mathrm{P} 1$, é um dado raro na literatura. Os estudos realizados até o momento só apresentaram padrões claros de controle por rejeição nos testes de equivalência quando o uso de diferentes proporções de $\mathrm{S}+\mathrm{e} \mathrm{S}$ - foi aliado a procedimentos adicionais que manipularam a resposta de observação, por exemplo a dica atrasada (Johnson \& Sidman, 1993; Magnusson, 2002), máscara (Perez et al., 2019) ou MTS com observação requerida (Perez et al., 2015, 2020). Em Perez et al. (2019), o uso de diferentes proporções de S+e S- sem o auxilio de manipulações da resposta de observação resultou em falhas durante o treino de discriminação condicional e dados inconclusivos durante os testes de equivalência. No presente estudo, nenhuma manipulação da resposta de observação foi realizada de modo a preservar as medidas obtidas pelo rastreador de movimentos dos olhos, o que pode ter dificultado a obtenção de padrões típicos de rejeição durante a condição RJ para os participantes $\mathrm{P} 2, \mathrm{P} 3$ e P4.

No caso do participante $\mathrm{P} 2$, o excessivo número de sessões necessárias até que o critério de aprendizagem fosse atingido parece fortalecer a hipótese de que, ao longo das tentativas de cada passo do treino, a participante aprendeu a selecionar cada um dos oito $\mathrm{S}+$ apresentados, em vez de rejeitar os dois S-. Os resultados referentes aos movimentos dos olhos do participante $\mathrm{P} 2$, em conjunto com os resultados dos testes, também dão suporte a essa hipótese; na condição RJ, para esse participante, foi alta a ocorrência de escolhas realizadas após observação exclusiva de $\mathrm{S}+$ (o oposto do que ocorreu com a participante P1). Para os participantes $\mathrm{P} 3$ e P4, a rápida aquisição do responder condicional durante o treino da segunda condição (SL) parece apontar o estabelecimento de um learning set (Saunders \& Spradlin, 1990). A aquisição do responder condicional tornou-se mais rápida (ocorreu com um menor número de tentativas) à medida que os participantes foram expostos a novas relações condicionais ao longo das condições.

Os resultados da condição LB mostram uma maior duração e frequência relativa de observação para o $\mathrm{S}+$. Tal resultado foi encontrado também por Huziwara et al. (2016; ver também Steingrimsdottir \& Arnsten, 2016). Embora tal diferença possa ser decorrente de uma redução da observação do S- ao longo das sessões, como observado em estudos anteriores (Steingrimsdottir \& Arnsten, 2016), a análise da estratégia de observação sugere que foram poucas as tentativas com escolhas realizadas após a observação de um único estímulo de comparação. Nesse caso, é difícil afirmar que o controle por seleção tenha sido majoritariamente estabelecido, especialmente considerando que estudos anteriores encontraram resultados positivos nos testes de equivalência a partir do estabelecimento simultâneo de ambos controles, por seleção e por rejeição (Arantes \& de Rose, 2015; de Rose et al., 2013). 
Os resultados da condição RJ da participante $\mathrm{P} 1$ mostram que o $\mathrm{S}$ - foi observado com maior duração e frequência do que o $\mathrm{S}+$ e que escolhas após a observação exclusiva do $\mathrm{S}$ foram mais frequentes do que após a observação exclusiva do $\mathrm{S}+$. Tal padrão de observação sugere o estabelecimento do controle por rejeição (Magnusson, 2002) e, por sua vez, contrasta com os resultados desse mesmo participante na condição SL, na qual o estímulo mais observado foi o S+ e escolhas após a sua observação exclusiva foram mais frequentes. A comparação dos resultados das condições RJ e SL mostra consistência entre o estabelecimento dos controles por seleção ou por rejeição informados pelos testes de equivalência e as medidas de observação. Assim, a observação maior do S- em comparação com o $\mathrm{S}+\mathrm{e}$ escolhas realizadas após a observação exclusiva do Ssugerem a ocorrência do controle por rejeição ao passo que a observação maior do $\mathrm{S}+$ em comparação com o S- e escolhas realizadas após a observação exclusiva do $\mathrm{S}+$ sugerem a ocorrência do controle por seleção. Nesse último caso, a comparação dos resultados de equivalência e observação foi especialmente regular na condição SL. O padrão observado para a participante $\mathrm{P} 1$ foi replicado, em geral, para a maioria dos participantes. Partindo dos resultados da condição SL, é possível interpretar os controles estabelecidos para os participantes P2, P3 e P4 na condição RJ, cujos resultados diferiram daqueles apresentados pela participante P1. Os resultados dos participantes P2 e P4 sugerem que o procedimento utilizado para estabelecer o controle por rejeição não impediu o estabelecimento de relações modelo-S+, como sugerido pela duração e frequência relativa de observação dos estímulos de comparação. Ainda, especialmente no caso do participante $\mathrm{P} 2$, foi possível observar uma alta ocorrência de escolhas realizadas após observação exclusiva do $\mathrm{S}+$. Os dados do participante P3, por outro lado, sugerem o estabelecimento de ambos controles, dada a ausência de qualquer diferenciação das medidas de observação para $\mathrm{S}+\mathrm{e} \mathrm{S}$ - ao longo das condições SL e RJ.

Estudos analítico-comportamentais que utilizaram rastreadores de movimentos oculares têm considerado a duração (Dube et al., 1999, 2003; 2010; Huziwara et al., 2015; 2016; Tomanari et al., 2007; Perez et al., 2015; Pessôa et al., 2009; Steingrimsdottir \& Arnsten, 2016), a frequência (Huziwara et al., 2016; Schroeder 1969, 1970, 1997; Steingrimsdottir \& Arnsten, 2016; Tomanari et al., 2007) e a estratégia (ou topografia) da observação (Dube et al., 2006; Endemann et al., 2011; Magnusson, 2002) como medidas auxiliares na avaliação do controle de estímulos. O presente estudo, entretanto, foi o primeiro a considerar as três medidas, conjuntamente, na avaliação do controle por seleção e por rejeição. Estudos futuros deveriam investigar as diferenças entre tais medidas, bem como em quais contextos cada uma delas deve ser utilizada. É provável que medidas referentes à estratégia ou topografia do olhar sejam mais adequadas para análises moleculares, i.e., na avaliação do controle intratentativa (e.g., Magnusson, 2002). Análises de duração e frequência absolutas ou relativas ao longo das sessões parecem se adequar como medidas molares, intertentativas (e.g. Huziwara et al., 2016; Perez et al., 2015; Steingrimsdottir $\&$ Arnsten, 2016), que revelam o controle prevalente em diferentes momentos do procedimento.

Por fim, como estratégia para evitar a perda das medidas oculares, o presente estudo reduziu o tamanho dos estímulos pela metade, em centímetros. Embora não seja possível uma comparação direta com os estudos realizados anteriormente (Huziwara et al., 2016; Magnusson, 2002), foram praticamente inexistentes as tentativas em que escolhas foram realizadas sem que pelo menos um dos estímulos de comparação fosse observado. Em estudos anteriores, com estímulos maiores e de mesma luminância (Pessôa et al., 2009), foi verificado, ao final do treino discriminativo, uma alta frequência de respostas de escolha sem registro de observação prévia dos estímulos mostrados na tela. A perda do registro não significa que os estímulos não tenham sido observados. Discute-se que as respostas aos estímulos apresentados, devido ao efeito da prática, passaram a ocorrer via visão periférica, tornando dispensável que os estímulos sejam fixados mais próximos ao centro da retina. Recomenda-se, assim, o uso de estímulos menores em pesquisas de controle de estímulos que envolvam o uso de rastreadores oculares. O uso do mouse, embora interfira no registro da observação, também pode ser uma alternativa para ampliar a duração da observação dos estímulos durante o treino discriminativo (Huziwara et al., 2016).

O presente estudo tem como principal conclusão o fato de que o estabelecimento dos controles por seleção e por rejeição são acompanhados de mudanças nas medidas de observação dos estímulos. Enquanto o controle por seleção tende a ser seguido de uma maior observação relativa de $\mathrm{S}+$ e de respostas de escolha baseadas em sua observação exclusiva, o estabelecimento do controle por rejeição tende a ser acompanhado por uma maior observação relativa do S- e de respostas de escolha seguidas de sua observação exclusiva. Estudos futuros poderão utilizar tecnologias de rastreamento do olhar e softwares de análise de dados que permitam manipular a ocorrência desses controles durante o treino e exigir, por exemplo, uma maior tempo de observação dos estímulos S+ ou S-, a depender do controle a ser favorecido. Tal tecnologia também teria importância em situações de aplicação, nas quais controles espúrios usualmente dificultam a aprendizagem das relações condicionais programadas pelo experimentador (e.g., Dube et al., 2010). 


\section{REFERÊNCIAS}

Arantes, A., \& de Rose, J. C. (2015) High probability of stimulus class formation with both sample-S+ and sample-S- controlling relations in baseline. The Psychological Record, 63, 743-748. doi:10.1007/s40732-015-0143-2

Carrigan, P. F., \& Sidman, M. (1992). Conditional discrimination and equivalence relations: A theoretical analysis of control by negative stimuli. Journal of the Experimental Analysis of Behavior, 58, 183-204. doi: 10.1901/jeab.1992.58-183

de Rose, J. C. (1996). Controlling factors in conditional discriminations and tests of equivalence. Em T. R. Zentall \& P. M. Smeets (Eds.), Stimulus class formation in humans and animals (pp. 253-277). Amsterdam: North Holland.

de Rose, J. C., \& Bortoloti, R. (2007). A equivalência de estímulos como modelo de significado. Acta Comportamentalia, 15, 83-102.

de Rose, J. C., Hidalgo, M., \& Vasconcelos, M. (2013). Controlling relations in baseline conditional discrimination as determinants of stimulus equivalence. The Psychological Record, 63, 85-98. doi: 10.11133/j.tpr.2013.63.1.00

Dixon, M. H., \& Dixon, L. S. (1978). The nature of standard control in children's matching-to-sample. Journal of the Experimental Analysis of Behavior, 30, 205-212. doi: 10.1901/ jeab.1978.30-205

Dube, W. V., Balsamo, L. M., Fowler, T. R., Dickson, C. A., Lombard, K. M., \& Tomanari, G. Y. (2006). Observing behavior topography in delayed matching to multiple samples. The Psychological Record, 56, 233-244. doi: 10.1007/BF03395547

Dube, W. V., Dickson, C. A., Balsamo, L. M., O’Donnell, K. L., Tomanari, G. Y., Farren, K. M., \& McIlvane, W. J. (2010). Observing behavior and atypically restricted stimulus control. Journal of the Experimental Analysis of Behavior, 94, 297-313. doi: 10.1901/jeab.2010.94-297

Dube, W. V., \& Hiris, J. (1999). Match to Sample Program (Version 11.6.7) [computer software]. Walthan, MA: E. K. Shriver Center of Mental Retardation.

Endemann, P., Pessôa, C. V. B. B., Perez, W. F., \& Tomanari, G. Y. (2011). Identificação de operantes verbais constituintes da leitura por meio da análise dos movimentos dos olhos. Psicologia: Teoria e Pesquisa, 27, 139-148. doi: 10.1590/ S0102-37722011000200016.

Huziwara, E. M., de Souza, D. G., \& Tomanari, G. Y. (2016). Patterns of eye movements in matching-to-sample tasks. Psicologia: Reflexão e Crítica, 29(2). doi: 10.1186/s41155016-0010-3

Huziwara, E. M., Silva, M. J. M., Perez, W. F., \& Tomanari, G. Y. (2015). Eye-movement behavior in simple simultaneous and successive discrimination tasks. Psicologia: Reflexão e Crítica, 28, 603-612. doi: 10.1186/s41155-016-0010-3

Johnson, C., \& Sidman, M. (1993). Conditional discrimination and equivalence relations: Control by negative stimuli. Journal of the Experimental Analysis of Behavior, 59, 333-347. doi: 10.1901/jeab.1993.59-333

Magnusson, A. (2002). Topography of eye movements under select and reject control. Dissertação de Mestrado, Shriver Center, Northeastern University, Boston.

McIlvane, W. J., Kledaras, J. B., Munson, L. C., King, K. A., de Rose, J. C., \& Stoddard, L. T. (1987). Controlling relations in conditional discrimination and matching by exclusion. Journal of the Experimental Analysis of Behavior, 48, 187-208. doi: 10.1901/jeab.1987.48-187

McIlvane, W. J., Serna, R., Dube, W. V., \& Stromer, R. (2000). Stimulus control topography coherence and stimulus equivalence: Reconciling test outcomes with theory. Em J. Leslie \& D. E. Blackman (Eds.), Issues in experimental and applied analysis of human behavior (pp. 85-101). Reno: Context Press.
McIlvane, W. J., Withstandley, J. K., \& Stoddard, L. T. (1984). Positive and negative stimulus relations in severely retarded individuals' conditional discrimination. Analysis and Intervention in Developmental Disabilities, 4, 235-251. doi: 10.1016/0270-4684(84)90003-X

Perez, W. F., Endemann, P., Pessôa, C. V. B., \& Tomanari, G. Y. (2015). Assessing stimulus control in a discrimination task with compound stimuli: Evaluating testing procedures and tracking eye fixations. The Psychological Record, 65, 83-88. doi: 10.1007/s40732-014-0092-1.

Perez, W. F., Huziwara, E. M., Rodrigues, R. M., Vilela, E. C., Tomanari, G. Y., \& Vaidya, M. (2020). Effect of observing response requirements to sample and comparison stimuli on the establishment of reject control (sample/S-relations). Journal of the Experimental Analysis of Behavior, 114, 60-71. doi: 10.1002/jeab.602

Perez, W. F., \& Tomanari, G. Y. (2008). Controles por seleção e rejeição em treinos de discriminação condicional e testes de equivalência. Revista Brasileira de Análise do Comportamento, $4,175-190$

Perez, W. F., \& Tomanari, G. Y. (2013). Inferindo a ocorrência dos controles por seleção e por rejeição em tarefas de emparelhamento com o modelo: uma revisão metodológica. Acta Comportamentalia, 21, 211-225.

Perez, W. F., \& Tomanari, G. Y. (2014). Indução dos controles por seleção e por rejeição em tarefas de emparelhamento com o modelo. Acta Comportamentalia, 22, 227-242.

Perez, W. F., Tomanari, G. Y., \& Vaidya, M. (2015). Effects of select and reject control on equivalence class formation and transfer of function. Journal of the Experimental Analysis of Behavior, 104, 146-166. doi:10.1002/jeab.164

Perez, W. F., Tomanari, G. Y., \& Vaidya, M. (2017). Effects of heterogeneous controlling relations on tests of transitivity and equivalence: An exploratory study. European Journal of Behavior Analysis, 18, 251-263. doi: 10.1080/15021149.2017.1309957

Perez, W. F., Vaidya, M., Huziwara, E. M., \& Tomanari, G. Y. (2019). Empirical analysis of biasing procedures to establish reject control relations. European Journal of Behavior Analysis, 114(1), 60-71. https://doi.org/10.1080/15021149.2019.1677 079

Pessôa, C., Huziwara, E., Perez, W. F., Endemann, P., \& Tomanari, G. Y. (2009). Eye fixations to figures in a four-choice situation with luminance balanced areas: Evaluating practice effects. Journal of Eye Movement Research, 2(3). doi: 10.16910/ jemr.2.5.3

Saunders, K. J., \& Spradlin, J. E. (1990). Conditional discrimination in mentally retarded subjects: Programming acquisition and learning set. Journal of the Experimental Analysis of Behavior, 60, 571-585. doi: 10.1901/jeab.1990.54-239

Steingrimsdottir, H. M., \& Arntzen, E. (2016). Eye movements during conditional discrimination training. The Psycological Record, 66, 201-212. doi: 10.1007/s40732-015-0156-x

Sidman, M. (1994). Equivalence relations and behavior: A research history. Boston: Authors Cooperative, INC. Publishers.

Sidman, M., \& Tailby, W. (1982). Conditional discrimination vs. matching to sample: An expansion of the testing paradigm. Journal of the Experimental Analysis of Behavior, 37, 5-22. doi: 10.1901/jeab.1982.37-5

Stromer, R., \& Osborne, J. G. (1982). Control by adolescent's arbitrary matching-to-sample by positive and negative stimulus relations. Journal of the Experimental Analysis of Behavior, 37, 329-348. doi: 10.1901/jeab.1982.37-329

Tomanari, G. Y., Balsamo, L. M., Fowler, T. R., Farren, K. M., \& Dube, W. V. (2007). Manual and ocular observing behavior in human subjects. European Journal of Behavior Analysis, 8 , 29-40. doi: 10.1080/15021149.2007.11434271 